\title{
FISH analysis in addition to G-band karyotyping: Utility in evaluation of myelodysplastic syndromes?
}

\author{
Wendy Yang, Brie Stotler, Deborah W. Sevilla, Foxwell N. Emmons, Vundavalli V. Murty, \\ Bachir Alobeid, Govind Bhagat*
}

Department of Pathology, Columbia University Medical Center, New York Presbyterian Hospital, New York, NY, USA

\section{A R T I C L E I N F O}

\section{Article history:}

Received 31 July 2009

Received in revised form 2 September 2009

Accepted 10 September 2009

Available online xxx

\section{Keywords:}

Myelodysplastic syndrome (MDS)

Karyotype

Giemsa banding

Fluorescence in situ hybridization (FISH)

\begin{abstract}
A B S T R A C T
Cytogenetic analysis provides important diagnostic and prognostic information for patients with myelodysplastic syndromes (MDS). Prior studies, mostly comprised of small sample sizes, have reported conflicting results while evaluating the usefulness of FISH in addition to G-band karyotyping in MDS. In the current study, the utility of performing a tailored FISH panel, in addition to G-band karyotyping was evaluated in a series of 110 MDS patients diagnosed at our institute. Using our FISH panel, clonal cytogenetic abnormalities were detected in 3/8 (38\%) of MDS cases with karyotype failure and in 5/54 (9\%) cases with normal G-band karyotypes, all the latter had intermediate or high grade MDS. Of the cases with abnormal G-band karyotypes, 6/48 (13\%) showed discrepancies between FISH and G-band results, however, FISH analysis only lead to reassignment of karyotypic abnormalities to different chromosomes, MDS cytogenetic risk stratification was not altered. Our findings suggest that FISH testing is informative only in MDS cases with karyotype failure and intermediate-high grade MDS cases with normal G-band karyotype and has limited utility in cases that have normal G-band karyotypes and morphologic features of low grade MDS or in cases with abnormal G-band karyotypes.
\end{abstract}

(c) 2009 Elsevier Ltd. All rights reserved.

\section{Introduction}

Myelodysplastic syndromes (MDS) are clonal stem cell disorders characterized by cytopenias, dysplasia in one or more cell lineages, and ineffective hematopoiesis. They are associated with significant morbidity and mortality due to bone marrow failure or evolution to acute myeloid leukemia, the latter occurring in $20-40 \%$ of patients [1]. Clonal chromosome abnormalities are detected in $40-60 \%$ of primary (de novo) MDS [2] and in $>90 \%$ of secondary (therapy-related) MDS cases [3] by Giemsa-band (Gband) karyotyping. Common recurrent cytogenetic abnormalities detected at diagnosis include, in order of decreasing frequency, $-7 / 7 q-,-5 / 5 q-,+8,20 q-,-Y, i(17 q)$ or $t(17 p),-13 / 13 q-$, $11 \mathrm{q}-, 12 \mathrm{p}-$ or $\mathrm{t}(12 \mathrm{p})$, with the most common abnormalities $(-7 / 7 q-,-5 / 5 q-,+8$ and $20 q-)$ occurring in about $40 \%$ of all MDS cases [4]. Cytogenetic analysis is an integral part of disease evaluation and assists not only in establishing the diagnosis, but also in establishing prognosis as codified by the Interna-

\footnotetext{
* Corresponding author at: Department of Pathologe, College of Physicians and Surgeons, Columbia University, VC14-228, 630W 168th Street, 14th Floor, New York, NY 10032, USA. Tel.: +1 212342 1323; fax: +1 2123052301.

E-mail address: gb96@columbia.edu (G. Bhagat).
}

tional Myelodysplastic Syndrome Working Group [5]. Three major risk categories have been defined based on the specific type(s) of cytogenetic abnormalities: (i) good risk-normal karyotype, isolated $5 \mathrm{q}-$, isolated $20 \mathrm{q}-$ and $-\mathrm{Y}$; (ii) poor risk-complex chromosome abnormalities (3 or more abnormalities), abnormalities of chromosome 7, and (iii) intermediate risk-all other cytogenetic abnormalities [4]. More recently, the presence of a monosomal karyotype has been suggested as an indicator of poor prognosis in acute myeloid leukemia [6]. It remains to be seen whether similar abnormalities also have prognostic relevance in MDS.

A variety of methodologies are currently utilized to detect chromosome abnormalities in human malignancies, including Gband karyotyping, fluorescence in situ hybridization (FISH), and the newer higher resolution techniques of comparative genomic hybridization (CGH) and single nucleotide polymorphism (SNP)array analyses [7]. Each of these techniques has specific advantages and disadvantages [7-9]. Prior studies have indicated that G-band karyotyping is a superior method of evaluating chromosome abnormalities in MDS compared to FISH analysis alone [10,11]. Moreover, FISH is a labor intensive and time consuming procedure. A few studies, evaluating the utility of FISH in addition to G-band karyotyping in the context of MDS, reported conflicting results; however, most were comprised of small sample sizes [3,9-15]. In this study, we 
evaluated the results obtained using a standard FISH panel in addition to G-band karyotyping in 110 cases of MDS to determine the usefulness of FISH analysis in three distinct settings, namely cases with G-band karyotype failure, cases with normal G-band karyotypes, and cases with abnormal G-band karyotypes.

\section{Materials and methods}

2.1. Case selection

We searched our departmental database to identify bone marrow (BM) biopsies and aspirates from individuals clinically suspected of having MDS that had been submitted for pathologic and cytogenetic evaluation at our institute over a 6-year period (January 2003-December 2008). Only cases that had BM biopsies and aspirates available for morphologic review and flow cytometric analysis were included in this study. Data regarding clinical findings and laboratory tests, including peripheral blood counts, were obtained from our laboratory information system. Clinical and laboratory evaluation used to exclude reactive and neoplastic disorders mimicking MDS, included, but was not restricted to, clinical examination and work-up for inflammatory (including autoimmune) and infectious disorders, hematopoietic and non-hematopoietic malignancies, congenital or acquired hemolytic anemias, hemoglobinopathies, coagulation disorders, and vitamin deficiencies, history of medication use or occupational exposure to toxins, flow cytometric analysis for paroxysmal nocturnal hemoglobinuria, FISH to detect BCRABL translocations, and polymerase chain reaction based analysis for JAK2 V617F mutations (only over the past 2 years) where indicated, imaging studies to rule out organomegaly, and peripheral blood cell counts to evaluate type and extent of cytopenias. Patients with elevations of any peripheral blood hematopoietic cell lineage were excluded from study. For pediatric patients, acquired and congenital forms of BM failure syndromes were also excluded by mutational analysis for Shwachmann-Diamond syndrome and chromosome breakage analysis for Fanconi anemia, as indicated.

\subsection{Morphologic evaluation, immunohistochemical staining, and flow cytometry}

Hematoxylin and Eosin (H\&E) stained sections of Bouin's-fixed, paraffinembedded BM biopsies and Wright-Giemsa stained aspirate smears were used for morphologic review, and iron stained aspirate smears and Wright-Giemsa stained peripheral blood smears, if available, were also evaluated. All cases were classified according to the WHO/FAB criteria $[4,16]$. Immunohistochemical staining was performed after heat-induced antigen retrieval according to standard methods and the primary antibodies used included CD34, CD117, CD61, myeloperoxidase, and glycophorin-A (DAKO, Carpinteria, CA). Four-color flow cytometric analysis was performed on BM aspirate specimens (FACScan; BD Bioscience, San Jose, CA, USA) using a comprehensive panel of monoclonal antibodies to analyze T-lineage $(C D 2, C D 3, C D 4, C D 5, C D 7, C D 8$, T-cell receptors alpha-beta and gamma-delta), B-lineage (CD19, CD20, CD22, CD79a, CD10, FMC7, surface and cytoplasmic kappa and lambda light chains), NK- or NK-T-lineage (CD16, CD56, and CD57 where indicated), erythroid lineage (glycophorin-A), and myeloid lineage (CD34, CD117, CD13, CD33, CD64, CD14, CD11c, MPO) specific antigens (BD Bioscience). Data were analyzed with Cell Quest software (BD Bioscience).

\subsection{Karyotype and fluorescence in situ hybridization (FISH) analysis}

All study cases underwent G-band karyotyping and FISH panel analysis. Gbanding of metaphase preparations was performed using standard methods and the karyotypes were described according to ISCN 2005 [17]. FISH was performed on methanol-acetic acid fixed cells according to standard protocols and the panel of probes used targeted the most common recurrent chromosome abnormalities $(-7 / 7 q-,-5 / 5 q-,+8$ and $20 q-)$; EGR1/D5S23 probes for chromosome 5q31and 5p15.2, D7S486/CEP7 probes for chromosome 7q31 and chromosome 7 centromere, D20S108 probe for chromosome 20q11, and CEP8 probe for chromosome 8 centromere (VYSIS, Downers Grove, IL, USA). 200-500 cells were analyzed and fluorescence signals were captured after counterstaining with DAPI using the Cytovision Imaging system attached to a Nikon Eclipse 600 microscope (Applied Imaging, Santa Clara, CA, USA).

To establish normal hybridization and variation controls for each of the probes used (EGR1/D5S23, D7S486/CEP7, CEP8, and D20S108), the probes were hybridized to interphase nuclei on preparations obtained from blood cultures of karyotypically normal males and females (5 cases each). At least 3 investigators scored the FISH signal patterns of qualifying interphase nuclei. These data were used to calculate the normal cut-off values for the detection of abnormal clones with a 95\% confidence level using the beta inverse function, as described [18]. Normal variations, established for each of the probes, were as follows: EGR1/D5S23 to determine del(5q), $0.8 \%$; D7S486/CEP7 to determine monosomy 7 or $\operatorname{del}(7 q)$, $0.8 \%$; D20S108 to determine $\operatorname{del}(20 \mathrm{q}), 2.4 \%$; and CEP8 to determine trisomy 8 , $0 \%$.
Table 1

MDS classification of 110 patients.

\begin{tabular}{lc}
\hline MDS classification & \# of patients \\
\hline RCUD & 19 \\
RCMD & 58 \\
RAEB-1 & 10 \\
RAEB-2 & 20 \\
MDS, NOS & 3 \\
Total & 110 \\
\hline
\end{tabular}

\subsection{Statistical analysis}

Statistical analyses including the Fisher's Exact Test were performed using the SAS statistical software, version 9.1 (SAS Institute, Inc., Cary, NC), $p$-values $<0.05$ were deemed significant.

\section{Results}

A total of 254 consecutive patients (144 males, 110 females, age range at diagnosis 3 months to 94 years, median age -44 years), whose BM specimens were sent for MDS work-up due to a clinical suspicion of MDS were identified during the study period. A diagnosis of MDS was established in 110 patients (65 males, 45 females, age range at diagnosis 11 months to 94 years, median age -65 years). The remaining 144 patients were deemed not to have MDS, since the cytopenias were eventually ascribed to underlying hematologic malignancies $(n=73)$, non-hematologic malignancies $(n=13)$, non-MDS bone marrow failure syndromes $(n=55)$ or hemoglobinopathies $(n=3)$. The MDS classification of all 110 patients is shown in Table 1 . Primary MDS was diagnosed in 102 patients and 8 had secondary MDS; 43/102 (42\%) of primary MDS patients had chromosomal abnormalities detected by G-band karyotyping, while $41 / 102(40 \%)$ of the patients had abnormalities detected by the MDS FISH panel of probes. Overall, $50 \%$ of the primary MDS patients (51/102) were found to have chromosomal abnormalities by G-band karyotyping and/or MDS FISH panel testing. Of the patients with secondary MDS, 5/8(63\%) had an abnormal karyotype by G-band analysis and 7/8 (90\%) had abnormalities detected by FISH.

The 110 MDS patients were divided into three subgroups based on G-band karyoptype results. The distribution of the types of MDS grades among each of these subgroups is shown in Table 2.

\subsection{Subgroup A (patients \#1 to \#54, Table 3): 54 MDS patients with normal G-band karyotypes}

This subgroup consisted of 34 males and 20 females (age range at diagnosis -11 months to 94 years, median age -48 years) and comprised 18 pediatric patients (age range 11 months to 16 years) and 36 adult patients (age range -20 years to 94 years). In this group, 5/54 individuals (9\%) had chromosomal abnormalities detected by MDS FISH panel testing (3 RCMD, 1 RAEB- 1 and 1 RAEB-2). Trisomy 8 was found in $3 / 5$ (60\%) of these patients in $2.4 \%$, $4 \%$ and $29 \%$ of cells, who were diagnosed with RCMD, RAEB- 1 and RCMD, respectively; 7q31 deletion was found in 29\% of the cells of a patient with RAEB-2, and monosomy 7 was found in $12 \%$ of the cells of a patient with RCMD. None of the patients with low grade MDS in this subgroup (10/54 =18\%) had any abnormalities detected by FISH (Table 4) and none of the pediatric patients in this category had any FISH detectable abnormality. Therefore, among only the patients with intermediate to high grade MDS in this subgroup, $5 / 44(11 \%)$ had abnormal FISH results. In addition, an increase in the percentage of blasts (1-19\%) in BM aspirate smears was noted more frequently in MDS patients with abnormal FISH results compared to those with normal FISH results $(4 / 5,80 \%$ vs. $8 / 49,16 \%$, $p=0.0068$ ). 
Table 2

Subgroups of MDS patients based on G-band karyotype status.

\begin{tabular}{lccc}
\hline & \multicolumn{2}{l}{ Karyotype status by G-band } & \\
\cline { 2 - 4 } & $\begin{array}{l}\text { Normal Karyotype } \\
\text { (subgroup A, } n=54)\end{array}$ & $\begin{array}{l}\text { Karyotype failure } \\
\text { (subgroup B, } n=8)\end{array}$ & $\begin{array}{l}\text { Abnormal Karyotype } \\
(\text { subgroup C, } n=48)\end{array}$ \\
\hline Number (\%) of low grade MDS & $10(19)$ & $5(63)$ & $7(15)$ \\
Number (\%) of intermediate grade MDS & $40(74)$ & $3(37)$ & $25(52)$ \\
Number (\%) of high grade MDS & $4(7)$ & $0(0)$ & $16(33)$ \\
\hline
\end{tabular}

Table 3

Subgroup A: MDS patients with normal G-band karyotypes.

\begin{tabular}{|c|c|c|c|c|}
\hline Patient & Age (years)/sex & Blast \% ${ }^{\mathrm{a}}$ & FISH & MDS subtype \\
\hline$\# 1$ & 11 months/M & 2 & Normal & RCMD \\
\hline \#2 & $12 / \mathrm{M}$ & 1 & Normal & RCMD \\
\hline \#3 & $10 / \mathrm{M}$ & 1 & Normal & RCUD \\
\hline$\# 4$ & $20 / F$ & 1 & Normal & RCMD \\
\hline \#5 & $1 / \mathrm{M}$ & 1 & Normal & RCMD \\
\hline \#6 & $1 / \mathrm{M}$ & 17 & Normal & RAEB-2 \\
\hline \#7 & $13 / \mathrm{M}$ & 1 & Normal & RCMD \\
\hline$\# 8$ & $3 / \mathrm{M}$ & 1 & Normal & RCMD \\
\hline \#9 & $66 / F$ & 1 & Normal & RCMD \\
\hline \#10 & $1 / \mathrm{M}$ & 1 & Normal & RCUD \\
\hline \#11 & $1 / F$ & 1 & Normal & RCMD \\
\hline \#12 & $5 / \mathrm{M}$ & 2 & Normal & RCUD \\
\hline \#13 & $11 / \mathrm{M}$ & 1 & Normal & RCMD \\
\hline \#14 & $65 / \mathrm{M}$ & 1 & Normal & RCMD \\
\hline \#15 & $72 / \mathrm{F}$ & 2 & Normal & RCMD \\
\hline \#16 & $57 / F$ & 1 & Normal & RCMD \\
\hline \#17 & $4 / \mathrm{M}$ & 1 & Normal & RCMD \\
\hline \#18 & $5 / \mathrm{F}$ & 1 & Normal & $2^{\circ} \mathrm{RCMD}$ \\
\hline \#19 & $73 / \mathrm{M}$ & 1 & Normal & RCUD \\
\hline \#20 & $53 / \mathrm{F}$ & 1 & Normal & RCMD \\
\hline \#21 & $85 / F$ & 1 & Normal & RCUD \\
\hline \#22 & $6 / \mathrm{M}$ & 1 & Normal & RCMD \\
\hline \#23 & $71 / \mathrm{F}$ & 1 & Normal & RCUD \\
\hline$\# 24$ & $52 / \mathrm{M}$ & 1 & Normal & RCMD-RS \\
\hline \#25 & $77 / \mathrm{M}$ & 1 & Normal & RCMD \\
\hline \#26 & $93 / \mathrm{M}$ & 1 & Normal & RCMD \\
\hline$\# 27$ & $74 / \mathrm{F}$ & 1 & Normal & RCMD \\
\hline \#28 & $4 / F$ & 1 & Normal & RCMD \\
\hline \#29 & $65 / \mathrm{M}$ & 1 & Normal & RCMD \\
\hline$\# 30^{\mathrm{b}}$ & $89 / \mathrm{M}$ & 3 & $8+(2.4 \%)$ & RCMD \\
\hline \#31 & $49 / F$ & 3 & Normal & RCMD \\
\hline$\# 32^{\mathrm{b}}$ & $80 / \mathrm{F}$ & 20 & $7 q-(29 \%)$ & RAEB-2 \\
\hline \#33 & $58 / \mathrm{M}$ & $<1$ & Normal & RCMD \\
\hline \#34 & $72 / \mathrm{M}$ & $<1$ & Normal & RCUD \\
\hline \#35 & $83 / \mathrm{F}$ & 1 & Normal & RCMD \\
\hline \#36 & $67 / F$ & 1 & Normal & RCMD \\
\hline \#37 & $94 / \mathrm{F}$ & 1 & Normal & RCMD \\
\hline \#38 & $11 / \mathrm{M}$ & 1 & Normal & RCMD \\
\hline \#39 & $70 / \mathrm{F}$ & 1 & Normal & RCUD \\
\hline \#40 & $65 / M$ & 1 & Normal & RCMD-RS \\
\hline \#41 & $78 / \mathrm{M}$ & 10 & Normal & RAEB-2 \\
\hline \#42 & $84 / \mathrm{M}$ & 1 & Normal & RCMD \\
\hline \#43 & $46 / F$ & 1 & Normal & RCMD \\
\hline$\# 44$ & $67 / M$ & $<1$ & Normal & RCMD \\
\hline \#45 & $52 / \mathrm{F}$ & 1 & Normal & RCUD \\
\hline$\# 46^{\mathrm{b}}$ & $73 / \mathrm{M}$ & 3 & $8+(29 \%)$ & RCMD \\
\hline \#47 & $16 / F$ & $<1$ & Normal & RCMD \\
\hline$\# 48$ & $6 / \mathrm{M}$ & 1 & Normal & RCMD \\
\hline \#49 & $59 / \mathrm{M}$ & 4 & Normal & RAEB-1 \\
\hline \#50 & $73 / \mathrm{M}$ & 13 & Normal & RAEB-2 \\
\hline$\# 51^{\mathrm{b}}$ & $68 / \mathrm{M}$ & 5 & $8+(4 \%)$ & RAEB-1 \\
\hline \#52 & $76 / \mathrm{M}$ & 1 & Normal & RCUD \\
\hline \#53 & $82 / \mathrm{M}$ & $<1$ & Normal & RCMD \\
\hline \#54 $4^{\mathrm{b}}$ & $62 / \mathrm{M}$ & 1 & $7-(12 \%)$ & $2^{\circ} \mathrm{RCMD}$ \\
\hline
\end{tabular}

Female: F; male: M.

a As determined by 500 cell count on bone marrow aspirate smear.

b Cases in which abnormalities were detected by FISH, but not by G-band karyotype.
Table 4

FISH in MDS patients with normal G-band karyotype (subgroup A) subdivided according to MDS grade.

\begin{tabular}{llc}
\hline & \multicolumn{2}{l}{ FISH status } \\
\cline { 2 - 3 } & Normal $(n=49)$ & Abnormal $(n=5)$ \\
\hline Number (\%) of low grade MDS & $10(20)$ & $0(0)$ \\
Number (\%) of intermediate grade MDS & $36(74)$ & $4(80)$ \\
Number (\%) of high grade MDS & $3(6)$ & $1(20)$ \\
\hline
\end{tabular}

\subsection{Subgroup B (patients \#55 to \#62, Table 5): 8 MDS patients with G-band karyotype failures.}

This subgroup consisted of 5 males and 3 females (age range at diagnosis -59 years to 87 years, median age -77 years), no pediatric cases were present in this category. In this subgroup $3 / 8$ individuals (38\%) had chromosomal abnormalities detected by FISH (1 RCUD and 2 RCMD). Deletion 20q11 was found in 1 patient (diagnosis - RCMD) in 78\% of the cells; trisomy 8 alone was found in 1 patient (diagnosis - RCMD) in $4 \%$ of the cells; and both trisomy 8 (6\% of cells) and $5 q 31$ deletion (5\% of cells) were found in 1 patient (diagnosis - RCUD). In this subgroup, there were no high grade MDS cases and none of the patients had a blast percentage $>1 \%$ on review of $\mathrm{BM}$ aspirate smears.

\subsection{Subgroup C (patients \#63 to \#110, Table 6): 48 MDS patients with abnormal G-band karyotypes}

This subgroup consisted of 26 males and 22 females (age range at diagnosis -4 years to 93 years, median age -60 years) and included 3 pediatric patients (age range -4 years to 15 years) and 45 adult patients (age range -18 years to 93 years). In this group $23 / 48$ individuals (47\%), all of whom were adults, had complex G-band karyotypes and in 14/48 individuals (29\%) the results of G-band karyotype and FISH were discordant, representing 2/3 (66\%) of pediatric and $12 / 45$ (27\%) of adult cases. However, further analysis revealed that in 8 of these 14 patients (57\%), including both of the pediatric patients, with abnormal G-band kayotypes but no FISH detectable abnormalities, the discrepancy was attributed to a failure of the FISH probes in targeting the chromosomal abnormalities observed on G-band karyotyping. On the other hand, in the remaining 6 of 14 patients (43\%) there was a true discrepancy between the results of G-band karyotype and FISH (differences

Table 5

Subgroup B: MDS patients with G-band karyotype failure.

\begin{tabular}{lllll}
\hline Patient & Age (years)/sex & Blast \% $^{\mathrm{a}}$ & FISH (\% abnormal cells) & MDS subtype \\
\hline$\# 55$ & $72 / \mathrm{M}$ & 1 & Normal & RCMD \\
$\# 56$ & $80 / \mathrm{M}$ & 1 & Normal & RCUD \\
$\# 57$ & $74 / \mathrm{F}$ & 1 & Normal & MDS, NOS \\
$\# 58$ & $82 / \mathrm{F}$ & 1 & Normal & RCUD \\
$\# 59$ & $59 / \mathrm{M}$ & 1 & $8+(4 \%)$ & RCMD \\
$\# 60$ & $83 / \mathrm{M}$ & 1 & Normal & RCUD \\
$\# 61$ & $87 / \mathrm{M}$ & 1 & $20 \mathrm{q}-(78 \%)$ & RCMD \\
$\# 62$ & $80 / \mathrm{F}$ & 1 & $5 q-(6 \%), 8+(5 \%)$ & $2 \circ$ RCUD \\
\hline
\end{tabular}

Female: F, male: $\mathrm{M}$

a As determined by 500 cell count on bone marrow aspirate smear. 


\begin{tabular}{|c|c|c|c|c|}
\hline Patient & Age $^{a} / \operatorname{sex}$ & MDS subtype & FISH (\% of abnormal cells) & Detailed karyotype \\
\hline \#63 & 8/M & RCMD & $7 q-(30 \%)$ & $46, \mathrm{XY}, \mathbf{d e l}(\mathbf{7})(\mathbf{q} 11.1)\{\mathbf{1 4}\} / 46, \mathrm{XY}\{6\}$ \\
\hline \#64 & $85 / \mathrm{F}$ & RCMD & $5 q-(5 \%)$ & $46, \mathrm{XX}, \operatorname{del}(\mathbf{5})(\mathbf{q} 14 \mathrm{q} 33)\{3\} / 46, \mathrm{XX}\{19\}$ \\
\hline \#65 & 71/M & RAEB-2 & $7-(23 \%)$ & $43, \mathrm{XY}, \operatorname{del}(5)(\mathrm{q} 13),-7, \mathrm{i}(8)(\mathrm{q} 10),-12,-17,-21,-22,+\operatorname{mar} 1-2\{10\} / 46, \mathrm{XY}\{6\}$ \\
\hline \#66 & $62 / \mathrm{F}$ & RAEB-1 & $5 q-(37.5 \%)$ & $41-47, \mathrm{XX}, \mathrm{t}(4 ; 15)(\mathrm{q} 12 ; \mathrm{p} 11.2),-5,-8,-11,-13,-15,-17,-19, \operatorname{add}(19)(\mathrm{p} 13), \operatorname{del}(20)(\mathrm{q} 11.2),+\mathrm{r}(?),+\operatorname{mar} 1-7\{\mathrm{cp} 21\} / 46, \mathrm{XX}\{3\}$ \\
\hline \#67 & 63/M & RCMD & $7 q-(4.7 \%)$ & $46, X Y, \operatorname{del}(7)(q 11.2)\{2\} / 46, X Y\{18\}$ \\
\hline \#68 & $93 / \mathrm{F}$ & RCMD & $5 q-(45 \%)$ & $44, \mathrm{XX}, \operatorname{der}(1) \operatorname{dup}(1)(\mathrm{q} 21 \mathrm{q} 43) \operatorname{ins}(1 ; ?)(\mathrm{p} 13 ; ?) \mathrm{t}(1 ; 1)(\mathrm{p} 32 ; \mathrm{q} 23),-5, \operatorname{del}(7)(\mathrm{p} 15), \operatorname{add}(7)(\mathrm{q} 36),-9,-15,+\operatorname{mar} 1\{13\} / 46, \mathrm{XX}\{2\}$ \\
\hline \#69 & 18/M & RAEB-1 & $7-(34 \%)$ & $45, X Y,-7\{20\}$ \\
\hline \#70 & $55 / \mathrm{M}$ & RCMD & $20 q-(76 \%)$ & $46, X Y, \operatorname{del}(\mathbf{2 0})(\mathbf{q 1 2})\{\mathbf{1 8}\} / 46, \mathrm{XY}\{2\}$ \\
\hline \#71 & $4 / \mathrm{F}$ & RCUD & MDS panel negative & $47, \mathrm{XX},+21\{15\}$ \\
\hline \#72 & $51 / \mathrm{F}$ & $2^{\circ}$ RCUD & $5 q-(54 \%), 7 q-(21 \%), 8+(24 \%)$ & 46,XX,del(5)(q23q31) $\{\mathbf{2}\} / 46, \mathrm{XX}, \operatorname{del}(\mathbf{5})(\mathbf{q} 23 q 31),-\mathbf{7},+\mathbf{8}\{6\} / 45, \mathrm{XX}, \operatorname{der}(5) \mathrm{t}(5 ; 6)(\mathrm{q} 32 ; \mathrm{q} 12),-6, \operatorname{del}(7)(\mathrm{p} 12 \mathrm{p} 15)\{6\} / 46, \mathrm{XX}\{6\}$ \\
\hline \#73 & $63 / \mathrm{F}$ & RAEB-2 & $5 q-(90 \%)$ & $46, \mathrm{XX}, \operatorname{del}(\mathbf{5})(\mathbf{q} 22 q 34)$, add $(17)(\mathrm{p} 12)\{14\} / 44, \mathrm{XX}, \operatorname{del}(\mathbf{5})(\mathbf{q} 22 \mathbf{q 3 4}), \mathrm{t}(11 ; 13)(\mathrm{p} 15.5 ; \mathrm{q} 11),-16,-17,-18,+\operatorname{mar} 1\{4\} / 46, \mathrm{XX}\{2\}$ \\
\hline \#74 & $18 / \mathrm{M}$ & RCMD & $7-(68 \%)$ & $45, \mathrm{XX},-7\{15\} / 46, \mathrm{XX}\{5\}$ \\
\hline \#75 & $71 / \mathrm{F}$ & MDS, NOS & MDS panel negative & $46, \mathrm{XX}, \mathrm{der}(6) \mathrm{t}(1 ; 6)(\mathrm{q} 21 ; \mathrm{p} 21.3)\{5\} / 46, \mathrm{XX}\{10\}$ \\
\hline \#76 & $62 / \mathrm{F}$ & MDS, NOS & $5 q-(86 \%), 7-(70 \%)$ & $46, \mathrm{XX}, \operatorname{del}(\mathbf{5})(\mathbf{q 1 2 q} 32)\{9\} / 46, \mathrm{XX}, \mathrm{idem},-7\{7\} / 46, \mathrm{XX}\{4\}$ \\
\hline \#77 & $61 / \mathrm{F}$ & $2^{\circ}$ RAEB-2 & $5-(64 \%), 5 q-(5 \%), 7-(47 \%)$ & $44-46, \mathrm{XX},-\mathbf{5}, \mathrm{t}(6 ; 11)(\mathrm{p} 21.3 ; \mathrm{q} 23),-7, \operatorname{add}(12)(\mathrm{p} 13)$, add $(15)(\mathrm{p} 11.2),-20,+\mathrm{r}(?)\{\mathrm{cp} 14\} / 46, \mathrm{XX}\{6\}$ \\
\hline \#78 & $42 / \mathrm{M}$ & $2^{\circ}$ RAEB-1 & $5 q-(54 \%), 7-(43 \%)$ & $44, \mathrm{XY},-\mathbf{5},-\mathbf{7}, \mathrm{add}(17)(\mathrm{p} 11.2)\{7\} / 44, \mathrm{XY}, \mathrm{idem}, \mathrm{del}(3)(\mathrm{p} 21)\{3\} / 46, \mathrm{XY}\{10\}$ \\
\hline \#79 & $18 / \mathrm{M}$ & $2^{\circ} \mathrm{RCMD}$ & $7-(84 \%)$ & $46, X X\{1\} / 45, X X,-7\{19\}$ \\
\hline \#80 & 76/M & RCMD & $20 q-(7 \%)$ & $45, X,-Y, \operatorname{del}(\mathbf{2 0})(\mathbf{q} 12)\{\mathbf{2}\} / 45, \mathrm{X},-\mathrm{Y}\{17\} / 46, \mathrm{XY}\{1\}$ \\
\hline \#81 & 66/M & RAEB-2 & $5 q-(77 \%), 20 q-(77 \%)$ & $\begin{array}{l}\text { 44-45,X,-Y,i(5)(p10),t(4;6)(q27;q27),+6,+9,del(11)(q23),-17,-18,-20, } \\
\operatorname{der}(21) \mathrm{t}(8 ; 21)(\mathrm{q} 11.2 ; \mathrm{p} 11.2), \pm \operatorname{mar} 1-2\{\mathrm{cp} 16\} / 45, \mathrm{X},-\mathrm{Y}, \mathrm{idem}, \operatorname{ins}(12 ; ?)(\mathrm{q} 13 ; ?)\{2\} / 46, \mathrm{XY}\{2\}\end{array}$ \\
\hline \#82 & $57 / \mathrm{M}$ & RAEB-2 & $7-(29 \%)$ & $45, \mathrm{XY}, \mathrm{add}(1)(\mathrm{p} 36), \mathrm{t}(3 ; 17)(\mathrm{q} 26 ; \mathrm{q} 23),-7, \mathrm{t}(15 ; 20)(\mathrm{p} 11.2 ; \mathrm{q} 11.2)\{\mathrm{cp} 4\} / 46, \mathrm{XX}\{16\}$ \\
\hline \#83 & $68 / \mathrm{M}$ & $2^{\circ}$ RAEB-1 & $7-(37 \%)$ & $45, X Y,-7\{8\} / 46, X Y\{12\}$ \\
\hline \#84 & 77/M & RAEB-2 & MDS panel negative & $47, \mathrm{XY}, \mathrm{del}(12)(\mathrm{p} 12.2 \mathrm{p} 13.3),+13, \mathrm{i}(17)(\mathrm{q} 10)\{21\}$ \\
\hline \#85 & $85 / \mathrm{M}$ & RCMD & $8+(15 \%)$ & $47, \mathrm{XY},+8\{\mathbf{8}\} / 46, \mathrm{XY}\{19\}$ \\
\hline \#86 & 53/M & RCMD & $5 q-(11 \%), 7-(14 \%), 8+(9 \%)$ & $44, \mathrm{XY},-3, \mathbf{d e l}(\mathbf{5})(\mathbf{q} 13),-7, \operatorname{add}(8)(\mathrm{p} 23),+\mathbf{i}(\mathbf{8})(\mathbf{q 1 0}),-12,-15,-17, \operatorname{der}(19) \mathrm{t}(19 ; ? ; 12)(\mathrm{p} 13.3 ; ? ; \mathrm{q} 13),+\operatorname{mar} 1-2\{12\} / 47, \mathrm{XY},+\mathrm{Y}\{8\}$ \\
\hline \#87 & $57 / \mathrm{M}$ & RAEB-2 & $7-(62 \%)$ & $46, \mathrm{XY}, \mathrm{t}(3 ; 17)(\mathrm{q} 26 ; \mathrm{q} 23)\{11\} / 45, \mathrm{XY}, \mathrm{idem},-\mathbf{7}\{\mathbf{6}\} / 46, \mathrm{XY}\{3\}$ \\
\hline \#88 & $15 / \mathrm{F}$ & RCMD & MDS panel negative & $46, \mathrm{X}, \mathrm{t}(\mathrm{X} ; 19)(\mathrm{p} 11.1 ; \mathrm{q} 13.1)\{9\} / 46, \mathrm{XX}\{11\}$ \\
\hline \#89 & $21 / \mathrm{F}$ & RCMD & $8+(16.5 \%)$ & $47, \mathrm{XX},+\mathbf{8}\{\mathbf{6}\} / 46, \mathrm{XX}\{14\}$ \\
\hline \#90 & 73/M & REAB-2 & $8+(5 \%)$ & $47, \mathrm{XY},+\mathbf{8}\{\mathbf{5}\} / 45, \mathrm{X},-\mathrm{Y}\{1\} / 46, \mathrm{XY}\{14\}$ \\
\hline \#91 & 77/M & RAEB-2 & $8+(3 \%)$ & $48, \mathrm{XY}, \operatorname{der}(?) \mathrm{t}(1 ; ?)(\mathrm{q} 21 ; ?)\{11\} / 46, \mathrm{XY}\{11\}$ \\
\hline \#92 & $83 / \mathrm{F}$ & RCUD & MDS panel negative & $46, \mathrm{XX}, \operatorname{del}(5)(\mathrm{q} 22 \mathrm{q} 33)\{3\} / 46, \mathrm{XX}\{20\}$ \\
\hline \#93 & 89/M & RCMD & $20 q-(11 \%)$ & $46, \mathrm{XY}, \operatorname{del}(\mathbf{2 0})(\mathbf{q 1 1 . 2})\{\mathbf{5}\} / 46, \mathrm{XY}\{15\}$ \\
\hline \#94 & $80 / \mathrm{F}$ & RCUD & $20 q-(61 \%)$ & $46, X X, \operatorname{del}(20)(q 11.2 q 13.1)\{20\} / 46, X X\{19\}$ \\
\hline \#95 & $60 / \mathrm{F}$ & RAEB-2 & $5 q-(81 \%), 20 q-(85 \%)$ & $\begin{array}{l}\text { 44-45,XX,-3,del(5)(q11.1q33),-10,der(12)t(12;17)(p13;q12),-13,der (15)hsr(p11.2), } \\
\operatorname{der}(16) \mathrm{t}(13 ; 16)(\mathrm{q} 14 ; \mathrm{q} 24),-17, \operatorname{del}(\mathbf{2 0})(\mathbf{q 1 1 . 1}),+21,+22,+1-3 \operatorname{mar}\{\operatorname{cp} 19\} / 46, \mathrm{XX}\{2\}\end{array}$ \\
\hline \#96 & $84 / \mathrm{F}$ & RCMD & MDS panel negative & $45, X,-X\{20\}$ \\
\hline \#97 & 74/M & RCUD & $8+(50 \%)$ & $46, X,-Y,+8\{13\} / 45, X,-Y\{7\}$ \\
\hline \#98 & 71/M & RCMD & MDS panel negative & $46, \mathrm{XY}, \mathrm{t}(6 ; 9)(\mathrm{p} 23 ; \mathrm{q} 24)\{17\} / 46, \mathrm{XY}\{3\}$ \\
\hline \#99 & $72 / \mathrm{F}$ & RAEB-2 & $8+(27 \%)$ & $47, \mathrm{XX},+8\{11\} / 46, \mathrm{XX}\{3\} / 92, \mathrm{XXXX}\{6\}$ \\
\hline \#100 & $88 / \mathrm{M}$ & RAEB-2 & $5 q-(30 \%), 7-(4 \%), 8+(28 \%), 20+(28 \%)$ & $55-56, \mathrm{XY},+1,+2, \operatorname{der}(3) \mathrm{t}(3 ; 15)(\mathrm{q} 12 ; \mathrm{q} 15), \operatorname{del}(\mathbf{5})(\mathbf{q} 31),+6,-\mathbf{7},+\mathbf{8},+9,+11,+13, \operatorname{add}(16)(\mathrm{q} 22),-17,+19,+20,+22,+\mathrm{r}(?),+\operatorname{mar} 1\{\mathrm{cp} 11) / 46, \mathrm{XY}\{9\}$ \\
\hline \#101 & 73/M & RAEB-1 & $5 q-(43 \%)$ & $44, \mathrm{XY}, \mathrm{t}(5 ; 12)(\mathrm{p} 13 ; \mathrm{p} 13.3), \operatorname{del}(\mathbf{5})(\mathbf{q} 22), \operatorname{der}(6) \operatorname{add}(6)(\mathrm{p} 23) \operatorname{ins}(6 ; ?)(\mathrm{q} 13 ; ?), \operatorname{add}(14)(\mathrm{q} 32),-15,-18\{\mathrm{cp} 18\} / 46, \mathrm{XY}\{2\}$ \\
\hline \#102 & $60 / \mathrm{F}$ & RAEB-1 & $5 q-(26 \%), 7-(21 \%)$ & $46, \mathrm{XX},-\mathbf{5},+\operatorname{mar} 1\{4\} / 45, \mathrm{XX}, \mathrm{idem},-\mathbf{7}\{\mathbf{1 1}\} / 46, \mathrm{XX}\{5\}$ \\
\hline \#103 & $82 / \mathrm{M}$ & RAEB-2 & $5 q-(36 \%), 7 q-(37 \%), 20 q-(34 \%)$ & $43-45, \mathrm{XY},-\mathbf{5}, \mathbf{d e l}(\mathbf{7})(\mathbf{q} 22 \mathrm{q} 34),-17, \operatorname{del}(\mathbf{2 0})(\mathbf{q 1 1 . 2}),+1 \operatorname{mar}\{\operatorname{cp} 10\} / 46, \mathrm{XY}\{1\}$ \\
\hline \#104 & $65 / \mathrm{F}$ & RCMD & MDS panel negative & $46, \mathrm{XX}, \operatorname{del}(13)(\mathrm{q} 13 \mathrm{q} 21.3)\{2\} / 46, \mathrm{XX}\{18\}$ \\
\hline \#105 & $74 / \mathrm{M}$ & RAEB-1 & $5 q-(78 \%)$ & 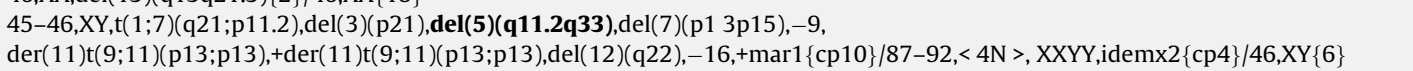 \\
\hline \#106 & $52 / \mathrm{M}$ & RAEB-2 & $7 q-(63 \%)$ & 46-47,XY,del(4)(q21q31.2),del(7)(q22q31),-17,+1-2mar\{cp13\}/46,XY $\{1\}$ \\
\hline \#107 & $81 / \mathrm{F}$ & RAEB-1 & MDS panel negative & $45, \mathrm{X},-\mathrm{X}\{4\} / 46, \mathrm{XX}\{16\}$ \\
\hline \#108 & $74 / \mathrm{F}$ & RAEB-2 & $7 q-(58 \%), 8+(45 \%)$ & $47, \mathrm{XX},+\operatorname{der}(1) \mathrm{t}(1 ; 7)(\mathrm{p} 11 ; \mathrm{p} 11.2),-\mathbf{7},+\mathbf{8}\{\mathbf{6}\} / 46, \mathrm{XX}\{14\}$ \\
\hline \#109 & $33 / \mathrm{F}$ & RAEB-2 & $7 q-(85 \%)$ & 46,XX,inv(3)(q21q26),del(7)(q22.1q34)\{2\}/46,idem,i(21)(q10)\{17\}/4 7,XX,idem,i(21)(q10)x2\{1\} \\
\hline \#110 & $48 / \mathrm{F}$ & RCMD & $5 q-(11 \%), 8+(21 \%), 20 q-(80 \%)$ & $41-44, \mathrm{XX},-7,-12,-18,-20,-21,-1 \backslash \mathrm{R} \backslash 3 \operatorname{mar}\{\mathrm{cp} 9\} / 46, \mathrm{XX}\{11\}$ \\
\hline
\end{tabular}

Bold reflects abnormalities detected by FISH panel. Female: $\mathrm{F}$ male: $\mathrm{M}$.

a Age given in years. 
were in the regions specifically targeted by the MDS FISH panel probes, Table 6). The frequency of complex karyotypes was higher, though not statistically significant, in these patients $(5 / 6,83 \%)$ compared to the patients with no discrepancy between $\mathrm{G}$-band analysis and FISH panel testing $(18 / 42,43 \%)(p=0.0912)$. Only 1 of 6 patients with discrepant G-band and FISH findings had a simple karyotypic abnormality (isolated deletion $5 q$ ) detected by $\mathrm{G}$-band analysis. FISH failed to detect this abnormality due to the deletion on the $5 q$ region being outside the region of coverage of the FISH probe.

\section{Discussion}

G-band karyotyping and FISH are the most commonly used methods to detect chromosomal abnormalities in MDS. Each method has specific advantages and disadvantages. G-band karyotyping allows analysis of all chromosomes to detect any abnormalities, however, it requires culture of viable cells to obtain metaphase spreads, which limits its ability to interrogate nonviable cells (i.e. archived material) or to detect abnormalities in clones with a low mitotic index and in minor neoplastic clones [9]. In addition, G-band karyotyping is also limited by its resolution power, which is in the range of at least a few mega-bases of DNA [8]. Consequently, G-band karyotyping cannot always establish the identity of marker chromosomes, addition of chromosome material, especially in complex rearrangements, or ring chromosomes. Thus, cryptic and/or sub-microscopic chromosome aberrations can be missed or incorrectly assigned to certain chromosomes or chromosome regions $[8,10]$. In MDS cases with complex abnormal karyotypes, G-band karyotyping may also miss chromosome abnormalities beyond its threshold of resolution [10]. On the other hand, FISH analysis is not limited by the viability of cells and it can be performed on non-dividing (interphase) cells [9]. FISH also has a much higher resolution power and can detect chromosomal aberrations in the order of a few hundred kilobases of DNA [8]. Therefore, FISH is quantitatively superior and more sensitive in detecting chromosomal aberrations in clones with low mitotic index, in detecting cryptic or sub-microscopic chromosomal aberrations, and in detecting minor clones. However, FISH analysis has limitations because only specific targeted chromosomal regions (to which the FISH probes bind) can be evaluated and chromosomal aberrations involving other regions cannot be examined [10,11]. It has been well established from prior studies that G-band karyotyping alone is, overall, a superior method of evaluating chromosomal abnormalities in MDS compared to FISH analysis alone. Previous studies showed that G-band karyotyping detected chromosomal abnormalities in 11-40\% MDS cases with negative FISH results, due to a failure to target the aberrant regions by FISH probes $[10,11]$. Our study (subgroup C) showed a frequency $(8 / 48,17 \%$ ) within the range observed previously for patients that had chromosome abnormalities detected by G-band karyotyping, but with "normal" FISH results, which was also attributed to a failure of the probes to target the cytogenetic aberration. Thus, our findings support the use of G-band karyotyping as the first line method for MDS cytogenetic evaluation.

Even though MDS patients with normal karyotypes belong to the good risk category, as defined by the International Myelodysplastic Syndrome Working Group, these patients constitute a biologically heterogeneous group with unpredictable outcomes $[11,14,19]$. Detection of chromosome abnormalities by FISH may help to stratify the prognostic risk profiles of patients within this subgroup $[12,14]$. Prior studies evaluating the utility of FISH in cases of MDS with normal karyotype have yielded contradictory results. In both studies, by Rigolin et al. [14] and Bernasconi et al. [12], a significant number of MDS patients (18\% and 15\%, respectively) with normal G-band karyotypes had abnormalities detected by FISH, which were also associated with a higher BM blast percentage, higher MDS grade, and worse prognosis. FISH was shown to detect cryptic or sub-microscopic chromosomal abnormalities and chromosome abnormalities in abnormal clones with low mitotic index, however, the particular types of abnormalities detected by FISH and their frequencies were different in these studies $[12,14,15]$. In contrast, other studies concluded that FISH was not able to detect chromosome abnormalities in a significant number of MDS cases with normal G-band karyotypes (0 to <5\%) [9-11,13]. In our study, 9\% of MDS patients with normal G-band karyotype (subgroup A) had abnormal FISH results. These patients all had intermediate or high grade MDS and the abnormal FISH results led to a re-categorization (upgrading) of their cytogenetic risk categories. This finding is consistent with the fact that higher grade MDS tend to have more frequent underlying cytogenetic abnormalities $[9,10,20]$. No low grade MDS cases with normal G-band karyotypes had abnormal FISH findings in our study. In conjunction with morphologic assessment, our results have implications for triaging cases for further genetic analysis, demonstrating that cases with low grade MDS and normal G-band karyotypes do not appear to benefit from FISH testing. Of the patients with normal G-band karyotypes and abnormal FISH results, the majority (60\%) had trisomy 8 , similar to the observations of Rigolin et al. [14]. Monosomy 7 detected by FISH in only one of our cases with normal G-band karyotypes was the predominant FISH abnormality reported in previous studies [12,15]. The discrepancy in the types and frequencies of abnormalities in the current and previous studies most likely reflects the small sample sizes of the latter $[12,15]$. The low frequency of abnormal clones detected by FISH in some patients with normal G-band karyotypes reflects the presence of subclones that likely had a low proliferative potential. Our findings confirm previous observations that MDS cases with normal G-band karyotypes and abnormal FISH results tend to have higher BM blast percentages compared to cases with normal G-band karyotypes and normal FISH results [12,14]. Of note, the vast majority of our pediatric patients had normal karyotypes, and similar to the adults, FISH testing did not uncover any abnormalities in this patient subgroup. The reasons are probably similar to the ones mentioned above, especially the failure of our FISH panel to detect genetic aberrations of pediatric MDS, which with the exception of monosomy 7 and trisomy 8 appear disparate from adults $[21,22]$.

One prior study has shown that FISH was able to detect chromosome abnormalities in a significant percentage (17\%) of MDS cases with G-band karyotype failure [15]. We observed a slightly higher frequency of cases with abnormal FISH findings (38\%) in this group (subgroup B), possibly due to the use of a more extensive FISH panel, supporting previous conclusions that FISH testing in such situations allows categorization of a significant number of MDS patients into the defined cytogenetic risk groups. FISH analysis in this subgroup detected only cytogenetic abnormalities belonging to the good (deletion 5q, deletion 20q) or intermediate (trisomy 8 ) risk categories. No poor prognosis abnormalities (e.g. involving chromosome 7) were identified. None of the patients in this subgroup had features of high grade MDS by morphologic criteria and none had $>1 \%$ blasts in their BM aspirates. These findings suggest that patients with G-band karyotype failure might have more indolent forms of MDS based on their cytogenetic abnormalities, MDS grade, and blast count.

The benefits of performing FISH analysis in cases of MDS with abnormal G-band karyotypes has been investigated previously $[10,11,15]$. FISH was able to identify the chromosomal origin of material gained or lost in certain chromosome aberrations that could not be resolved by G-band karyotyping alone, such as marker chromosomes, addition of material, ring chromosomes, or homogeneously staining regions [8-10]. FISH has also been shown to detect chromosome abnormalities masked by highly complex karyotypic 
abnormalities and to redefine chromosome abnormalities that were erroneous by G-band karyotype [8-10]. Overall, in previous studies, FISH analysis led to reassignment of cytogenetic abnormalities in 11-33\% of MDS cases with abnormal G-band karyotypes by identifying additional cryptic or sub-microscopic chromosome aberrations and by clarifying the origins of chromosome material in complex karyotypic aberrations [ $10,11,15]$. In our study, only $13 \%$ of patients with abnormal G-band karyotypes (subgroup C) displayed true discrepancies between the results of karyotype and FISH analyses. Pseudo discrepancies, consisting of abnormal G-band karyotypes and normal FISH results, were more frequent because the FISH panel used in our study did not target all of the chromosome regions with aberrations. Two of the three pediatric patients in this group displayed pseudodiscrepancies, since the FISH probes used were not directed against the cytogenetic abnormalities, while the case with del $7 q$ was identified correctly. Complex karyotypes were identified much more frequently in the patients with true discrepancies (83\%) compared to the rest of the patients in this subgroup (43\%). However, since complex karyotypes assigned patients to a poor cytogenetic risk category of MDS to begin with, the modification of chromosomal abnormalities after incorporation of FISH results in these patients did not alter their already poor cytogenetic risk status. Therefore, for the purposes of cytogenetic risk stratification per se, our results indicate no additional value of performing FISH in MDS cases with complex G-band karyotypes.

It needs to be noted that our study, like many of the previous reports, was of a retrospective nature, and thereby has inherent weaknesses. These are in part due to a reliance only on written records, as well as the possibility of differing stringencies, or non-uniform use, of clinical and laboratory parameters used by different physicians to exclude disorders, both neoplastic and non-neoplastic, which could mimic MDS, and the unavailability of certain molecular tests for older cases that are now used in the diagnostic work-up of patients suspected of having MDS e.g. testing for JAK2 mutations. Thus, our observations need to be validated in future prospective studies by other investigators to confirm their practical utility. Furthermore, due to the relatively low number of pediatric patients in our series, corroboration from larger studies of MDS in this patient group is warranted to confirm our results.

In summary, apart from the biologic implications, our findings have practical utility in the diagnostic work-up of MDS cases. We confirm prior observations that G-band karyotyping alone is a superior method of evaluating chromosome abnormalities in MDS compared to FISH analysis alone. Our data indicate that triage of specimens for FISH analysis is better facilitated after review of BM findings, including aspirate morphology. FISH analysis provides valuable information regarding the presence of cytogenetic abnormalities in cases of karyotype failure, which is required for categorization of MDS into the appropriate cytogenetic risk groups, in a significant number (38\%) of cases. With regard to MDS cases with normal G-band karyotypes, FISH testing should be considered for intermediate and high grade MDS, since cytogenetic abnormalities were only detected in these cases. Lastly, although FISH leads to a correction of the G-band karyotype results in a fraction of MDS cases with abnormal karyotypes, little is gained in terms of cytogenetic risk stratification from a clinical viewpoint as complex karyotypes by themselves connote poor outcomes.

\section{Conflict of interest statement}

Authors declare no conflicts of interest.

\section{Acknowledgements}

The authors wish to thank Garrick Cuman for his help in organizing the material for review and the histology laboratory at
Columbia University Medical Center for superb technical assistance.

Contributions: All authors made substantial contributions to the conception and design of the study, drafting and/or revising the article, and gave final approval of the submitted version.

\section{References}

[1] Aul C, Gattermann N, Schneider W. Epidemiological and etiological aspects of myelodysplastic syndromes. Leuk Lymphoma 1995;16:247-62.

[2] Parlier V, van Melle G, Beris P, Schmidt PM, Tobler A, Haller E, et al. Hematologic, clinical, and cytogenetic analysis in 109 patients with primary myelodysplastic syndrome. Prognostic significance of morphology and chromosome findings. Cancer Genet Cytogenet 1994;78:219-31.

[3] Vallespi T, Imbert M, Mecucci C, Preudhomme C, Fenaux P. Diagnosis, classification, and cytogenetics of myelodysplastic syndromes. Haematologica 1998;83:258-75.

[4] Swerdlow SHCEHN. In: Jaffe ES, Pileri SA, Stein H, Thiele J, Vardiman JW, editors. WHO classification of tumours of haematopoietic and lymphoid tissues. Lyon: IARC; 2008.

[5] Mufti GJ, Bennett JM, Goasguen J, Bain BJ, Baumann I, Brunning R, et al. Diagnosis and classification of myelodysplastic syndrome: International Working Group on Morphology of myelodysplastic syndrome (IWGM-MDS) consensus proposals for the definition and enumeration of myeloblasts and ring sideroblasts. Haematologica 2008;93:1712-7.

[6] Breems DA, Van Putten WL, De Greef GE, Van Zelderen-Bhola SL, GerssenSchoorl KB, Mellink CH, et al. Monosomal karyotype in acute myeloid leukemia: a better indicator of poor prognosis than a complex karyotype. J Clin Oncol 2008;26:4791-7.

[7] Speicher MR, Carter NP. The new cytogenetics: blurring the boundaries with molecular biology. Nat Rev Genet 2005;6:782-92.

[8] Cuneo A, Bigoni R, Cavazzini F, Bardi A, Roberti MG, Agostini P, et al. Incidence and significance of cryptic chromosome aberrations detected by fluorescence in situ hybridization in acute myeloid leukemia with normal karyotype. Leukemia 2002;16:1745-51.

[9] Kibbelaar RE, Mulder JW, Dreef EJ, van Kamp H, Fibbe WE, Wessels JW, et al. Detection of monosomy 7 and trisomy 8 in myeloid neoplasia: a comparison of banding and fluorescence in situ hybridization. Blood 1993;82: 904-13.

[10] Beyer V, Castagne C, Muhlematter D, Parlier V, Gmur J, Hess U, et al. Systematic screening at diagnosis of $-5 / \operatorname{del}(5)(q 31),-7$, or chromosome 8 aneuploidy by interphase fluorescence in situ hybridization in 110 acute myelocytic leukemia and high-risk myelodysplastic syndrome patients: concordances and discrepancies with conventional cytogenetics. Cancer Genet Cytogenet 2004;152:29-41.

[11] Cherry AM, Brockman SR, Paternoster SF, Hicks GA, Neuberg D, Higgins RR, et al. Comparison of interphase FISH and metaphase cytogenetics to study myelodysplastic syndrome: an Eastern Cooperative Oncology Group (ECOG) study. Leuk Res 2003;27:1085-90.

[12] Bernasconi P, Cavigliano PM, Boni M, Calatroni S, Klersy C, Giardini I, et al. Is FISH a relevant prognostic tool in myelodysplastic syndromes with a normal chromosome pattern on conventional cytogenetics? A study on 57 patients. Leukemia 2003;17:2107-12.

[13] Ketterling RP, Wyatt WA, VanWier SA, Law M, Hodnefield JM, Hanson CA, et al. Primary myelodysplastic syndrome with normal cytogenetics: utility of 'FISH panel testing' and M-FISH. Leuk Res 2002;26:235-40.

[14] Rigolin GM, Cuneo A, Roberti MG, Bardi A, Bigoni R, Piva N, et al. Exposure to myelotoxic agents and myelodysplasia: case-control study and correlation with clinicobiological findings. Br J Haematol 1998;103:18997.

[15] Romeo M, Chauffaille Mde L, Silva MR, Bahia DM, Kerbauy J. Comparison of cytogenetics with FISH in 40 myelodysplastic syndrome patients. Leuk Res 2002;26:993-6.

[16] Bennett JM, Catovsky D, Daniel MT, Flandrin G, Galton DA, Gralnick HR, et al. Proposed revised criteria for the classification of acute myeloid leukaemia. A report of the French-American-British Cooperative Group. Ann Intern Med 1985;103:620-5.

[17] Shaffer LGTNI. An international system for human cytogenetic nomenclature. Basel, Switzerland: Karger; 2009.

[18] Wolff DJ, Bagg A, Cooley LD, Dewald GW, Hirsch BA, Jacky PB, et al. Guidance for fluorescence in situ hybridization testing in hematologic disorders. J Mol Diagn 2007;9:134-43.

[19] West RR, Stafford DA, White AD, Bowen DT, Padua RA. Cytogenetic abnormalities in the myelodysplastic syndromes and occupational or environmental exposure. Blood 2000;95:2093-7.

[20] Greenberg P, Cox C, LeBeau MM, Fenaux P, Morel P, Sanz G, et al. International scoring system for evaluating prognosis in myelodysplastic syndromes. Blood 1997;89:2079-88.

[21] Niemeyer CM, Kratz CP. Paediatric myelodysplastic syndromes and juvenile myelomonocytic leukaemia: molecular classification and treatment options. Br J Haematol 2008;140:610-24.

[22] Sasaki H, Manabe A, Kojima S, Tsuchida M, Hayashi Y, Ikuta K, et al. Myelodysplastic syndrome in childhood: a retrospective study of 189 patients in Japan. Leukemia 2001;15:1713-20. 